\title{
Auto-adjunticidade não-linear e leis de conservação da equação de Korteweg-de Vries-Zakharov-Kuznetsov
}

\author{
Priscila L. da Silva e Igor L. Freire \\ CMCC - Centro de Matemática, Computação e Cognição, UFABC, \\ 09210-170, Santo André, SP \\ E-mail: priscila.silva@ufabc.edu.br \\ E-mail: igor.freire@ufabc.edu.br,
}

Resumo: Neste trabalho apresentaremos condições para que a equação de KdV-ZK seja nãolinearmente auto-adjunta e, assim, utilizaremos o Teorema de Ibragimov para encontrar leis de conservação dela.

Palavras-chave: Leis de conservação, Teorema de Ibragimov, Teorema de Noether

\section{Introdução}

Quando falamos em quantidades conservadas, geralmente estamos pensando em conservação de quantidades como energia, momento

Em meados do século $X X$, a alemã Emmy Noether mostrou que leis de conservação de equações diferenciais provenientes das equações de Euler-Lagrange são originadas de alguma propriedade de simetria da equação diferencial considerada. Tal resultado é conhecido na literatura como Teorema de Noether.

O Teorema de Noether, todavia, apesar de sua elegância e aplicabilidade, torna-se inutilizável ao considerarmos equações diferenciais que não venham das equações de Euler-Lagrange.

Em 2006, Ibragimov [3] introduziu uma possível generalização do Teorema de Noether para encontrar leis de conservação de equações diferenciais, sejam elas ordinárias ou parciais, independetemente de serem ou não equações de Euler-Lagrange, removendo, desta maneira, a maior restrição com respeito à aplicabilidade do Teorema de Noether.

Despretenciosamente falando, o que Ibragimov fez foi, a partir de uma equação (aqui restringirnos-emos a equações, mas poder-se-ia discutir, também, sobre sistemas), construir uma equação auxiliar, chamada equação adjunta. O sistema formado pela equação original e sua adjunta admite sempre uma lagrangeana, que é simples engenhosamente construído à partir da primeira equação. Dessa forma, o sistema está nas condições do Teorema de Noether e, então, pode-se construir, para o sistema, leis de conservação utilizando-se da teoria de Noether.

O grande "problema" em se utilizar o que Ibragimov propôs é que, no ato de construção da equação adjunta-se, surge uma nova variável, não constante na equação original. Tal variável faz-se presente nas leis de conservação construídas, de modo que elas são leis de conservação não-locais.

Entretanto, para certas classes de equações diferenciais, é possível transformar as leis de conservação não-locais em locais, veja $[2,3,4]$.

Recentemente, em [8], leis de conservação para a equação de Zakharov-Kuznetsov (KZ)

$$
u_{t}+\alpha u u_{x}+\beta u_{x x x}+\gamma u_{x y y}=0,
$$

onde $u=u(t, x, y)$ e $\alpha, \beta, \gamma$ são constantes reais, foram encontradas utilizando-se dos desenvolvimentos sugeridos por Ibragimov, uma vez que a equação (1) não é proveniente das equações de Euler-Lagrange. 
A equação (1) descreve o comportamento de ondas fracas não-lineares íon-acústicas no plasma magnetizado e elétrons isotérmicos na presença de um campo magnético uniforme, e foi deduzida por Zakharov e Kuznetsov em [10]. Para maiores detalhes, veja [5] e referências presentes. A equação (1) é uma generalização da equação de Korteweg-de Vries (KdV).

Neste trabalho consideraremos uma generalização de (1), chamada de Korteweg-de VriesZakharov-Kuznetsov (KdV-ZK), dada por

$$
u_{t}+\alpha u u_{x}+u_{x x x}+u_{x y y}+u_{x z z}=0
$$

com $u=u(t, x, y, z)$, e estudaremos, via Teorema de Ibragimov, suas leis de conservação.

\section{Auto-adjunticidade}

Considere uma equação diferencial não-linear

$$
F\left(x, u, u_{(1)}, \ldots, u_{(k)}\right)=0
$$

onde $x=\left(x^{1}, \ldots, x^{n}\right), u=u(x)$ e $u_{(j)}, 1 \leq j \leq k$ denota as derivadas de $u$ de ordem $j$.

Em $[3,2]$, Ibragimov definiu a equação adjunta de $F$, denotada por $F^{*}$, como sendo

$$
F^{*}=\frac{\delta}{\delta u} \mathcal{L}
$$

onde $\mathcal{L}=v F$ é chamada de Lagrangeana formal, $v$ é uma nova variável não-local e $\frac{\delta}{\delta u}$ é o operador de Euler-Lagrange dado pela soma formal

$$
\frac{\delta}{\delta u}=\frac{\partial}{\partial u}+\sum_{s=1}^{\infty}(-1)^{s} D_{i_{1}} \ldots D_{i_{s}} \frac{\partial}{\partial u_{i_{1}} \ldots u_{i_{s}}},
$$

com

$$
D_{i}=\frac{\partial}{\partial x^{i}}+u_{i} \frac{\partial}{\partial u}+u_{i j} \frac{\partial}{\partial u_{j}}+\ldots
$$

Aqui assumimos a notação de Einstein para índices repetidos.

Exemplo 1. A equação adjunta da equação é (2)

$$
v_{t}+\alpha u v_{x}+v_{x x x}+v_{x y y}+v_{x z z}=0
$$

pois, denotando $F=u_{t}+\alpha u u_{x}+u_{x x x}+u_{x y y}+u_{x z z}$, temos que

$$
F^{*}=-\left(v_{t}+\alpha u v_{x}+v_{x x x}+v_{x y y}+v_{x z z}\right) .
$$

Logo, a condição $F^{*}=0$ resulta na equação (5).

Em $[3,2]$ Ibragimov propôs a seguinte definição:

Definição 1. A equação diferencial (3) é dita ser estritamente auto-adjunta se existe função $\lambda=\lambda\left(x, u, u_{(1)}, \ldots, u_{(k)}\right)$ tal que

$$
\left.F^{*}\right|_{v=u(x)}=\lambda F .
$$

A definição anterior pode ser reformulada da seguinte forma: a equação diferencial (3) é dita ser estritamente auto-adjunta se a mudança $v=u$ na equação adjunta se anula identicamente nas soluções $u$ da equação original. 
Exemplo 2. A equação (2) é estritamente auto-adjunta, pois, de (6), temos

$$
\left.F^{*}\right|_{v=u}=-\left(u_{t}+\alpha u u_{x}+u_{x x x}+u_{x y y}+u_{x z z}\right) .
$$

Dessa forma $\left.F^{*}\right|_{v=u}$ se, e somente se, $F=0$.

Pensando em casos de equações que não eram estritamente auto-adjuntas e suas consequências em leis de conservação, Ibragimov em [4] propôs uma nova definição que contemplasse a Definição 1, mas que também englobasse outros casos de equações que não se encaixavam nela.

Definição 2. A equação diferencial (3) é dita ser não-linearmente auto-adjunta se existem funções $\phi=\phi(x, u)$ e $\lambda=\lambda\left(x, u, u_{(1)}, \ldots, u_{(k)}\right)$ tais que

$$
\left.F^{*}\right|_{v=\phi(x, u)}=\lambda F .
$$

A função $\phi(x, u)$ é chamada substituição.

Um dos principais resultados deste trabalho pode, agora, ser enunciado:

Teorema 1. A equação de $K d V-Z K$

$$
u_{t}+\alpha u u_{x}+u_{x x x}+u_{x y y}+u_{x z z}=0
$$

é não-linearmente auto-adjunta, com substituição dada por

$$
\phi(t, x, y, z, u)=a_{1}(\alpha t u-x)+a_{2} u+h(y, z),
$$

onde $h(y, z)$ é uma função arbitrária e $a_{1}, a_{2}$ são constantes.

Este teorema generaliza vários resultados recentes da literatura de equações não-linearmente auto-adjuntas, como mostram os corolários abaixo.

Corolário 1. A equação de $Z K(1)$ é não-linearmente auto-adjunta.

Demonstração. De fato, a equação (1) pode ser obtida da equação (9) impondo a condição $u_{z}=0$. Do ponto de vista da substituição, equivale a requerer independência com respeito a $z$. Requerindo isso na expressão (10), concluímos que a substituição para a equação (1) é $\phi(t, x, y, u)=a_{1}(\alpha t u-x)+a_{2} u+h(y)$.

O resultado acima nada mais é que uma prova alternativa para um dos resultados cruciais obtidos em [8].

Corolário 2. A equação de Korteweg-de Vries

$$
u_{t}+\alpha u u_{x}+u_{x x x}=0
$$

é não-linearmente auto-adjunta.

Demonstração. Notando que a equação (11) pode ser obtida a partir da equação (kdvzk) eliminando a dependência em $(y, z)$, exigindo que a substituição (10) satisfaça $\phi_{y}=\phi_{z}=0$, concluímos que $h(y, z)=a_{3}$. Dessa forma, a substituição para a equação $(11)$ é $\phi(t, x, u)=$ $a_{1}(\alpha t u-x)+a_{2} u+a_{3}$. 
Observamos que o Corolário 2 recupera os resultados obtidos em [1] com respeito à equação (11).

Na equação (9), $u$ tem como variáveis espaciais $x, y, z$. Numa generalização ao Teorema 1 , mostraremos que a condição (10) ainda se mantém suficiente, mas neste caso a função $h$ é dada por $h=h\left(y_{1}, \ldots, y_{n}\right), u=u(t, y)$, onde $y=\left(x, y_{1}, \ldots, y_{n}\right) \in \mathbb{R} \times \mathbb{R}^{n}$.

Neste caso, a equação de KdV-ZK se escreve

$$
u_{t}+\alpha u u_{x}+u_{x x x}+(\Delta u)_{x}=0
$$

em $(0, \infty) \times \mathbb{R} \times \mathbb{R}^{n}$, onde $\Delta$ é o Laplaciano

$$
\Delta=\frac{\partial^{2}}{\partial y_{1}^{2}}+\cdots+\frac{\partial}{\partial y_{n}^{2}}
$$

\section{Teorema de Ibragimov e leis de conservação}

O famoso Teorema de Noether é uma ferramenta importante para a obtenção de leis de conservação para equações diferenciais com princípios variacionais, isto é, proveniente das equações de Euler-Lagrange via alguma Lagrangeana.

Porém, por exemplo, a conhecida equação KdV (11) não provém das equações de EulerLagrange. Assim, o Teorema de Nother é incapaz de encontrar sequer uma lei de conservação. Sabe-se, todavia, que a equação ?? possui infinitas leis de conservação, veja [7, 9].

Tentando generalizar o Teorema de Noether, Ibragimov em [2] propôs o que ele chamou de "a new conservation theorem", chamado de Teorema de Ibragimov em [?]. Nesse novo teorema, considerando uma equação diferencial (3), possuindo ela Lagrangeana ou não, leis de conservação não-locais foram encontradas, via Teorema de Noether, para o sistema

$$
\left\{\begin{array}{l}
F=0 \\
F^{*}=0
\end{array} .\right.
$$

Para utilizar o Teorema de Noether, Ibragimov demonstrou em [3, 2] que as simetrias de Lie

$$
X=\xi^{i} \frac{\partial}{\partial x^{i}}+\eta \frac{\partial}{\partial u}
$$

admitidas por $F$ são herdadas pela sua adjunta $F^{*}$ e, além disso, a Lagrangeana formal $\mathcal{L}=v F$ é de fato uma Lagrangeana para o sistema (13).

Assim, o vetor de componentes

$$
C^{i}=\xi^{i} \mathcal{L}+W \frac{\delta \mathcal{L}}{\delta u_{i}}+\sum_{s \geq 1} D_{i_{1}} \ldots D_{i_{s}}(W) \frac{\delta \mathcal{L}}{\delta u_{i i_{1} \ldots i_{s}}},
$$

onde $W=\eta-\xi^{i} u_{i}, i=1, \ldots, n$, é uma quantidade conservada não-local para o sistema (13).

Entretanto, se a equação diferencial $F=0$ for não-linearmente auto-adjunta, então conseguimos eliminar a variável não local $v$ fazendo a substituição necessária para que $F=0$ seja não-linearmente auto-adjunta, veja [4]. Desta forma, o vetor com componentes (14) se torna uma lei de conservação local para a equação $F=0$.

Neste trabalho, além da classificação sobre auto-adjunticidade não-linear, apresentaremos leis de conservação para a equação $\operatorname{KdV-ZK~}(2)$ em $(0, \infty) \times \mathbb{R} \times \mathbb{R}^{2}$, utilizando o Teorema 1 , o Teorema de Ibragimov e resultados obtidos em $[5,6]$.

\section{Agradecimentos}

Os autores agradecem à FAPESP pelo suporte financeiro através dos processos 2011/19089-6 e 2012/22725-4. I. L. Freire agradece ao CNPQ, através do processo no 308941/2013-6. 


\section{Referências}

[1] I. L. Freire and J. C. S. Sampaio, Nonlinear self-adjointness of a generalized fifth-order KdV equation, J. Phys. A: Math. Theor., 45 (2012), 032001.

[2] N. H. Ibragimov, Integrating factors, adjoint equations and Lagrangians, J. Math. Anal. Appl., 318 (2006) 742-757.

[3] N. H. Ibragimov, A new conservation theorem, J. Math. Anal. Appl., 333 (2007) 311-328.

[4] N. H. Ibragimov, Nonlinear self-adjointness and conservation laws, J. Phys. A Math. Theor., vol. 44, 8pp., (2011).

[5] B. T. Matebese, A. R. Adem, C. M. Khalique and A. Biswas, Solutions of ZakharovKuznetsov equation with power law nonlinearity in $(1+3)$ dimensions, Phys. Wave Phenom., vol. 19, pp. 148-154, (2011).

[6] M. Nadjafikhah and F. Ahangari, Symmetry analysis and similarity reduction of the Korteweg-de Vries-Zakharove-Kuznetsov equation, Asian-European J. Math., 5 (2012) 1250006, 22 páginas.

[7] A. G. Rasin and J. Schiff, Infinitely many conservation laws for the discrete KdV equation, J. Phys. A: Math. Theor., vol. 42, 16 pp., (2009).

[8] R. Tracinà, On the nonlinear self-adjointness of the Zakharov-Kuznetsov equation, Commun. Nonlinear Sci. Numer. Simulat., vol. 19, pp. 377-382, (2014).

[9] H. Yang and X. Xu, Hamiltonian structure and infinite number of conservation laws for the coupled discrete KdV equations, Appl. Math. J. Chinese Univ. Ser. B , vol. 19, pp. 374-380, (2004).

[10] V. E; Zakharov and E. A. Kuznetsov, On three-dimensional solitons, Sov. Phys., vol. 39, pp. 285-286, (1974). 